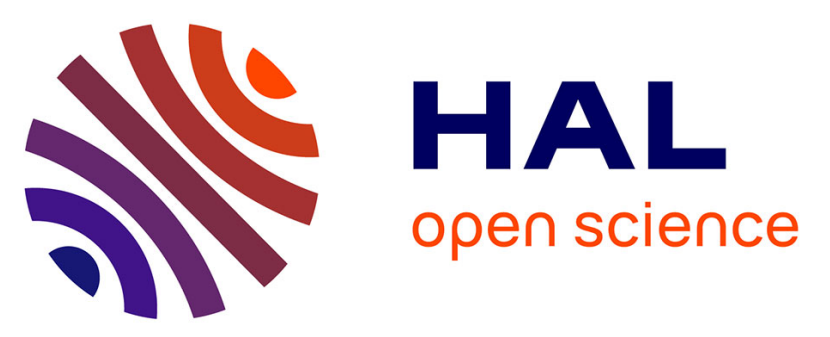

\title{
Mise en place du réseau viaire et des noms de rues sur la rive droite de Paris au cours du XIIIe siècle (à travers les livres d'archives de Saint-Magloire, Saint-Martin-des-Champs, Saint-Antoine-des-Champs, Saint-Eloi et du Temple)
}

Marlène Helias-Baron

\section{To cite this version:}

Marlène Helias-Baron. Mise en place du réseau viaire et des noms de rues sur la rive droite de Paris au cours du XIIIe siècle (à travers les livres d'archives de Saint-Magloire, Saint-Martin-des-Champs, Saint-Antoine-des-Champs, Saint-Eloi et du Temple). XVIIe Colloque d'Onomastique. Noms de lieux, noms de personnes: la question des sources. Toponymie urbaine de Paris et de sa banlieue, Dec 2015, Paris, France. halshs-01731574

\author{
HAL Id: halshs-01731574 \\ https://shs.hal.science/halshs-01731574
}

Submitted on 14 Mar 2018

HAL is a multi-disciplinary open access archive for the deposit and dissemination of scientific research documents, whether they are published or not. The documents may come from teaching and research institutions in France or abroad, or from public or private research centers.
L'archive ouverte pluridisciplinaire HAL, est destinée au dépôt et à la diffusion de documents scientifiques de niveau recherche, publiés ou non, émanant des établissements d'enseignement et de recherche français ou étrangers, des laboratoires publics ou privés. 


\section{Mise en place du réseau viaire et des noms de rues sur la rive droite de Paris au cours du XIII ${ }^{\mathrm{e}}$ siècle (à travers les livres d'archives de Saint-Magloire, Saint-Martin-des-Champs, Saint-Antoine-des-Champs, Saint-Eloi et du Temple) ${ }^{1}$}

Paris et ses rues, son urbanisation dans la Cité ainsi que sur les rives droite et gauche, ont été les objets de nombreuses études depuis le XVIII ${ }^{\mathrm{e}}$ siècle, plus ou moins pittoresques comme le Tableau de Paris de Louis-Sébastien Mercier ou la présentation par Alfred Franklin des rues et des cris de Paris au XIII ${ }^{\mathrm{e}}$ siècle $^{2}$, ou plus scientifiques comme les travaux de Simone Roux sur l'essor urbain de la rive gauche avec en arrière-plan «l'insertion réussie du grand établissement religieux qu'est l'abbaye Sainte-Geneviève dans la grande ville [et] la greffe efficace de l'institution universitaire sur l'organisme urbain $»^{3}$, ainsi que ceux de nombreux étudiants de l'École nationale des chartes comme Geneviève Etienne sur le Temple, Estelle Guerber sur le quartier de l'hôtel Saint-Paul aux XIV et XV siècles ou encore Noémie Escher sur Sainte-Geneviève ${ }^{4}$, sans oublier la magistrale étude de Valentine Weiss sur les cens et les rentes à Paris qui a permis de mieux cerner les contours des différentes censives de la capitale 5 .

Dans cette chaîne de travaux, mon étude se consacrera à la mise en place des rues et de leurs noms sur la rive droite de la fin du XII ${ }^{\mathrm{e}}$ au début du XIV siècle, mais elle restera partielle, faute d'avoir abordé les rôles de la taille ${ }^{6}$, ou encore les registres d'ensaisinement. Elle a néanmoins été menée à travers l'exploitation d'actes originaux, de cartulaires et de censiers. Son objectif est de mettre en évidence, si cela est possible, la structuration en rues de l'espace parisien ainsi que les interactions entre les religieux et la société parisienne, dans un contexte d'expansion démographique et urbaine principalement dans la partie de la ville que les sources parisiennes appellent « Outre-Grand-pont ». Selon le Dit des rues de Paris composé par le poète Guillot au début du XIV siècle, Paris intra-muros compte 310 rues $^{7}$, dont 184 pour la rive droite $^{8}$. Ainsi, à la recherche d'informations toponymiques et topographiques, j'ai d'abord exploité les cartulaires de Saint-Magloire, de Saint-Martin-des-Champs et de Saint-Antoinedes-Champs, puis les censiers de ces institutions, ainsi que ceux de Saint-Eloi et du Temple ${ }^{9}$, pour savoir dans quelle mesure leur organisation a été commandée par celle de l'espace parisien et quel a été le rôle de ces écrits dans la stabilisation des noms de rues à la fin du XIII ${ }^{\mathrm{e}}$ siècle. Ainsi, ces documents, par leur structuration interne, seraient le reflet d'un état de l'espace urbain, dont une partie est déjà organisée en rues et dont une autre est encore rurale ou, à tout le moins, en cours de transformation.

Dans cette étude, ce sont les rues qui importent, le bâti en revanche n'a pas été pris en compte, sauf pour servir de point de repère ${ }^{10}$. Par ailleurs, le choix des établissements religieux

\footnotetext{
${ }^{1}$ Comme les travaux se font rarement en solitaire, je tiens à remercier chaleureusement Sébastien Barret et Caroline Bourlet de leurs conseils et de leur attention toujours soutenue lors de la rédaction de cet article.

${ }^{2}$ MERCIER Louis-Sébastien 1781-1788. FRANKLIN Alfred 1874.

${ }^{3}$ RouX Simone 1989, citation issue du prologue du t. I, p. I.

${ }^{4}$ ETIENNE Geneviève 1974. GUERBER Estelle 2001. ESCHER Noémie 2009.

${ }^{5}$ WEISS Valentine 2009.

${ }^{6}$ BOURLET Caroline et POISSON Jean-François 2016

${ }^{7}$ BNF, ms. fr. 24432, f. $257 v^{\circ} \mathrm{a}-261$ vº b. MAREUSE Edgar (éd.) 1875. NiCOLAS Catherine (éd.) 2012.

${ }^{8}$ Guillot écrit 194 à la fin de son poème, mais il semblerait que ce soit une erreur de calcul de sa part. Voir NICOLAS Catherine 2012, p. 110 : «Guillot si fait a tous savoir que, par deça Grant-Pont, pour voir, n'a que .ii.c. rues mains sis ».

${ }^{9}$ Pour Saint-Antoine-des-Champs : Archives nationales, LL 1595 ; pour Saint-Martin-des-Champs : BNF, lat. 10977 et Archives nationales, LL 1351 ; pour Saint-Magloire : Archives nationales, LL 39 et BNF, lat. 5413 ; pour Saint-Eloi : Archives nationales, LL 75.

${ }^{10}$ DESCIMON Robert et WEISS Valentine 2012, p. 39 : «Les maisons des notables [...] formaient des points de repère qui structuraient la perception du paysage urbain ».
} 
présentés ci-dessus a été fait en fonction de leur complémentarité institutionnelle et territoriale, dans un espace compris à l'intérieur et à l'extérieur de l'enceinte de Philippe Auguste avant la construction de celle de Charles V, c'est-à-dire dans un territoire où la ville s'est étendue au cours du XIII ${ }^{\mathrm{e}}$ siècle.

Avant de commencer, il est nécessaire de préciser que l'étude se fonde sur une documentation encore dominée par le latin, puis par le bilinguisme, ce qui pose le problème de l'interprétation et de la traduction de certains termes utilisés en latin, notamment le mot vicus ${ }^{11}$, ainsi que celui de la fluctuation des dénominations des rues entre les différents documents, notamment en français ${ }^{12}$.

\section{La prise en compte du réseau viaire par les religieux dans leurs livres d'archives}

Dans les livres d'archives étudiés ici, on remarque que les religieux, lors de leur travail de composition, ont pris en compte les réalités urbaines et organisé les informations dont ils avaient besoin en fonction des rues. Ainsi, ces manuscrits apparaissent comme des reflets du réseau viaire intra-muros et de sa mise en place extra-muros.

Saint-Magloire, abbaye bénédictine d'abord installée dans la Cité, a été déplacée entre 1138 et 1159 sur la rive droite ${ }^{13}$, à proximité du marché aux Champeaux implanté peu de temps auparavant, en 1137. Ce déplacement s'accompagne du regroupement de la censive de l'abbaye en un bloc intra et extra-muros. Dans cet espace structuré par la présence du monastère mais déjà urbanisé au XIII ${ }^{\mathrm{e}}$ siècle, après une campagne très peu documentée de lotissement dans le bourg dans la seconde moitié du XII ${ }^{\mathrm{e}}$ siècle $^{14}$, l'accroissement de la population, et l'intensification de l'urbanisation qui en découle, est perceptible à travers le censier de $1274^{15}$. Ce document est inséré dans le Petit cartulaire, projet de cartularisation entrepris en 1294 à l'initiative de l'abbé, Louis II de Montfort (1273-1299). Resté à l'état d'embryon sans raison explicite, il a, semble-t-il, été remplacé lors de la rédaction à nouveau frais du Grand cartulaire en 1331 par Jean de Rozay, prévôt du monastère avant d'en devenir l'abbé (1332-1349) ${ }^{16}$. Outre ce censier, le Petit cartulaire contient un deuxième censier daté de 1294 d'après son incipit ${ }^{17}$.

À travers le premier censier écrit en latin avec quelques ajouts en français ${ }^{18}$, apparaissent les noms d'une quinzaine de rues situées au niveau de la Montagne Sainte-Geneviève, sur l'île

\footnotetext{
${ }^{11}$ Quand le mot vicus se trouve écrit dans les documents ici étudiés, faut-il le traduire systématiquement par « rue », sachant qu'il désigne d'abord le bourg ou le village ? Par ailleurs, dans les documents, la petite rue est désignée par le terme ruella (notamment dans les censiers de Saint-Martin-des-Champs ou dans le cartulaire de SaintAntoine-des-Champs). On trouve également, mais plus rarement calceya ou calceia (chaucié), pour désigner la chaussée d'une grande voie située en dehors des murailles (notamment la chaussée de la grande rue Saint-Martin ou celle de la grande rue Saint-Antoine dans les censiers de Saint-Martin-des-Champs, de Saint-Magloire ou de Saint-Eloi). Le terme strata enfin est utilisé en 1279 pour désigner une rue dans le diplôme de Philippe III le Hardi adressé au Temple (Archives nationales, K $34 \mathrm{n}^{\circ} 24$ ).

${ }^{12}$ Pour la géolocalisation et la cartographie, je me suis appuyée sur les données disponibles sur la plateforme Alpage qui est une plateforme d'information géo-historique sur Paris (AnaLyse diachronique de l'espace urbain PArisien : approche Géomatique) : http://alpage.huma-num.fr/fr/

13 Terroine Anne et Fossier Lucie 1998, t. 1, p. 14 et 518. HAMON Etienne 2012, p. 50.

${ }^{14}$ Terroine Anne et Fossier Lucie 1998, t. 1, p. 25-26.

${ }^{15}$ Ce censier occupe les quinze premiers feuillets du Petit cartulaire de Saint-Magloire.

${ }^{16}$ Petit cartulaire (Archives nationales, LL 39) et Grand cartulaire (Bibliothèque nationale de France, latin 5413). Terroine Anne et Fossier Lucie 1976, t. 3, p. VIII-IX. EAED. 1998b, p. 181-208. WeISS Valentine 2009, t. I, p. 538 et p. 629.

${ }^{17}$ Archives nationales, LL 39, f. $23 \mathrm{r}^{\circ}$ : « Cum secundum tempora moderna brevitate moderni gaudeant, idcirco tedio dispendii superflui primitus relegato ad comodum nostre ecclesie Sancti Maglorii Parisiensis et rerum ad eam pertinencium hunc librum fieri fecimus sub anno Domini $\mathrm{M}^{\circ} \mathrm{CC}^{\circ}$ nonagesimo quarto, in modum qui sequitur ».

${ }^{18}$ WeISS Valentine 2009, t. 2, p. 973. Archives nationales, LL 39, ff. $1 \mathrm{r}^{\circ}$ « a petit pont pour la meson maistre Richart lespicier $2 \mathrm{~s}$. » et $8 \mathrm{r}^{\circ}$. Le français s'impose à la fin du censier aux ff. $14 \mathrm{r}^{\circ}-15 \mathrm{r}^{\circ}$.
} 
de la Cité, puis, sur la rive droite, autour de Saint-Magloire et dans le Bourg l'Abbé en dehors des remparts, dans un espace compris entre la Grande rue Saint-Denis et la rue Saint-Martin, avec la rue Aubry-le-Boucher, la rue aux Oies, la rue du Bourg-l'Abbé (axe central du Bourg l'Abbé), Le Huleu, Darnestat et la rue Palée. Pour faciliter la consultation des informations, les noms des rues sont précédés d'un pied de mouche souvent rehaussé de rouge, signe utilisé fréquemment dans les livres d'archive à cet usage ${ }^{19}$, alors que les maisons de ceux qui doivent acquitter les cens sont introduites par un Item abrégé. Écrit à longues lignes d'une façon soignée, dans une écriture cursive régulière, le censier est structuré par termes (octave de Saint-Denis, Noël, Pâques, Nativité de saint Jean-Baptiste) et, à l'intérieur de ces parties, selon les rues dans lesquelles se trouvent les censitaires, avec l'amorce d'une sorte d'itinéraire.

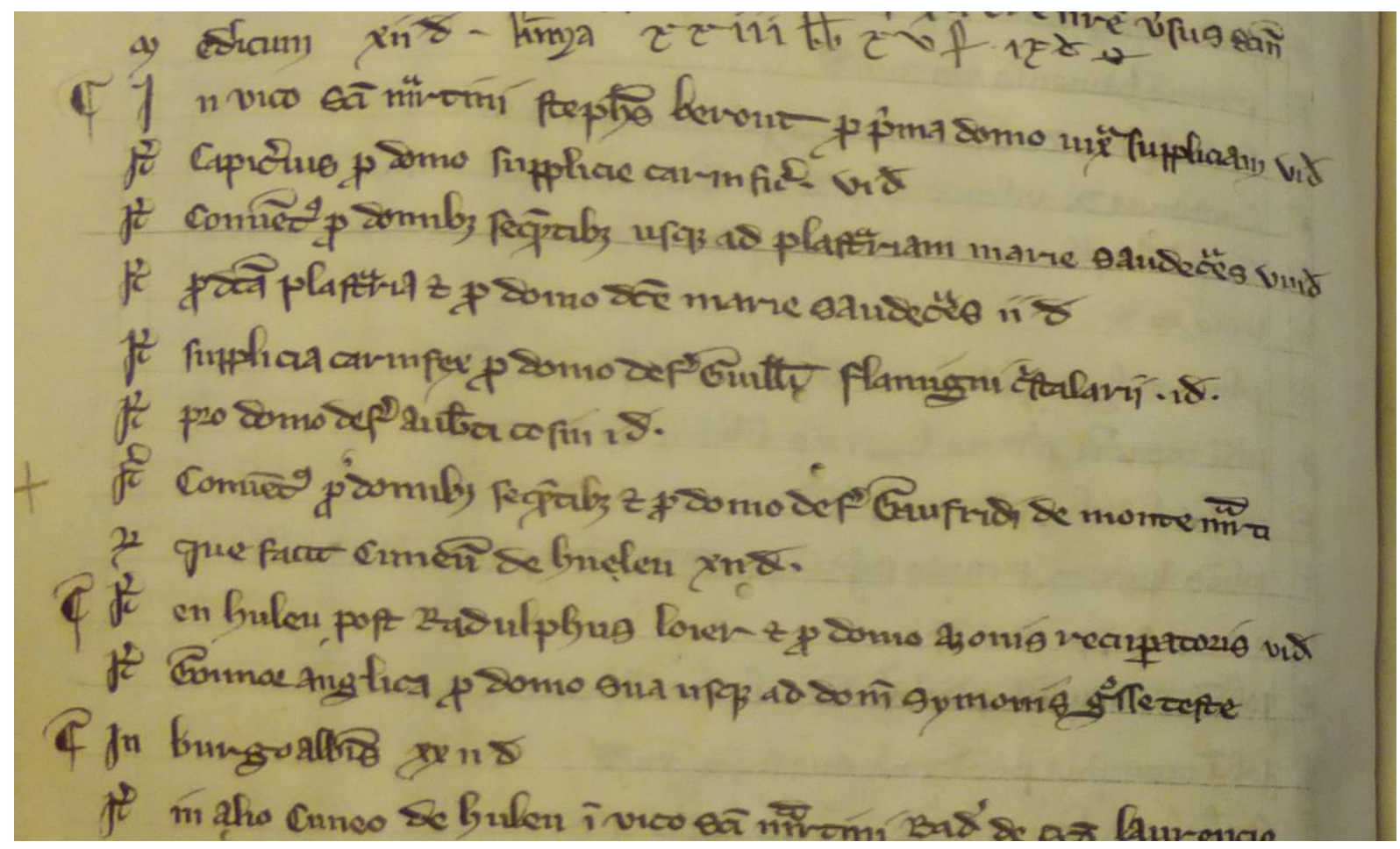

Figure 1 : Censier de Saint-Magloire, Arch. nat. LL39, utilisation de pieds de mouche pour signaler les rues Saint-Martin, Le Huleu et le Bourg l'Abbé (f. 3v)

Ce constat vaut également pour les censiers de Saint-Martin-des-Champs rédigés dans la deuxième moitié du XIII ${ }^{\mathrm{e}}$ siècle. Autour de cet établissement ${ }^{20}$, collégiale extra-parisienne devenue prieuré clunisien en 1079, situé au nord du rempart, un bourg extra-urbain s'est mis en place dès le $\mathrm{XI}^{\mathrm{e}}$ siècle. Le tracé des voies y est régulier, signe d'une intervention volontaire ${ }^{21}$. Ce prieuré dispose d'abord de deux cartulaires rédigés dans la première moitié du $\mathrm{XII}^{\mathrm{e}}$ et au début du XIII ${ }^{\mathrm{e}}$ siècle, ce qui correspond pour lui à un siècle de prospérité économique ${ }^{22}$. Ils ne sont toutefois d'aucune aide pour l'étude de la toponymie parisienne puisqu'ils concernent avant tout les possessions rurales de l'établissement. Ils semblent en fait ignorer la réalité urbaine, même si dans le cartulaire le plus tardif, quelques mentions de maisons parisiennes apparaissent pour les années 1200-1209. En revanche, les censiers des années 1263-1300 sont plus intéressants pour notre propos ${ }^{23}$. Valentine Weiss, dans sa thèse d'École des chartes, en a repéré

\footnotetext{
${ }^{19}$ BERTRAND Paul 2015, p. 184.

${ }^{20}$ MERCIER Alain 2012.

${ }^{21}$ TerRoINe Anne et FosSIER Lucie 1998, t. 1, p. 25.

${ }^{22}$ Liber testamentorum, Bibliothèque nationale, ms. lat. 10977 et Cartulaire A, Archives nationales, LL 1351.

${ }^{23}$ Archives nationales, LL 1378 : censiers de 1263, 1274, 1293 et 1300. Bibliothèque nationale, ms. lat. 10978 : censier de 1300. Voir: WeISS Valentine 1993, t. 4.
} 
deux pour le XIII ${ }^{\mathrm{e}}$ siècle ${ }^{24}$. Le premier est « la réunion de quatre censiers [de 1263, 1274, 1293 et 1300], probablement indépendants les uns des autres à l'origine, et reliés par la suite ». À chaque fois, les cens sont répartis entre les quatre termes habituels à Paris (Saint-Rémi, Noël, Pâques, Saint-Jean-Baptiste) et, à l'intérieur de ces parties, ils suivent un classement topographique par rues. Le deuxième recueil daté de 1300 adopte le même type de classement (par termes et par rues). Grâce à ces documents, la localisation de la censive de Saint-Martin se manifeste clairement : elle dessine deux espaces, un premier autour de l'abbaye et de son bourg à l'extérieur des remparts et un second à l'est des Halles dans Paris intra-muros, le long de la grande rue Saint-Martin, axe structurant de la censive désigné comme « magnus vicus Sancti Martini ». Intra-muros et dans le bourg l'Abbé, les cens énumérés portent sur des maisons situées le long des rues. Ces indications montrent bien que les possessions martiniennes se trouvent dans un espace fortement urbanisé ou en cours d'urbanisation. Néanmoins, au-delà du couvent des Filles-Dieu, vers Montmartre, apparaissent d'autres types de toponymes davantage liés aux activités rurales ou maraîchères, introduits notamment par l'emploi du terme maresia.

Cette prise en compte des réalités topographiques préexistantes est nécessaire pour les religieux, à la fois pour faciliter la consultation des écrits qu'ils ont produits et pour éviter les conflits avec leurs censitaires en cas d'ambiguïtés sur les toponymes. Pour le monastère de Saint-Antoine-des-Champs, cette adaptation semble être encore plus poussée. Fondé en dehors de Paris en 1204, à partir d'un établissement pour prostituées repenties créé à la fin du XII ${ }^{\mathrm{e}}$ siècle par le prédicateur Foulques de Neuilly ${ }^{25}$, il aurait été incorporé à l'ordre cistercien en $1208^{26}$. Il se situe à l'est du quartier Saint-Paul, sur la route de Meaux, donc dans un espace essentiellement rural au début du XIII ${ }^{\mathrm{e}}$ siècle. Malgré cette localisation marginale, les moniales ont acquis au cours du XIII ${ }^{\mathrm{e}}$ siècle de nombreuses maisons dans Paris, ainsi que les rentes qui pesaient sur elles. L'importance de leurs possessions parisiennes est perceptible dans leur cartulaire $^{27}$, exactement contemporain du Dit des rues de Paris du poète Guillot ${ }^{28}$.

Le manuscrit montre que les moniales, en partie issues des grandes familles bourgeoises parisiennes $^{29}$, connaissent parfaitement la topographie et la toponymie de Paris, même s'il est probable que son rédacteur ne soit pas l'une d'entre elles. Sa structure repose en effet sur la topographie parisienne et non sur une conception cistercienne de l'espace. On y trouve les noms d'une vingtaine de rues $^{30}$, localisées autour de certains points bien identifiés du paysage

\footnotetext{
${ }^{24}$ WeISS Valentine 1993, t. I, p. 2-15.

${ }^{25}$ Delaforge-Marchand Sandrine 1994, t. 1, p. 1.

${ }^{26}$ Archives nationales, L $1015 \mathrm{n}^{\circ}$ 3. L'acte d'incorporation de Saint-Antoine-des-Champs pose un problème de datation. Catherine Marchal, en étudiant cet acte, a relevé des incohérences au niveau des initiales des cinq abbés qui ne correspondent pas aux abbés en charge en 1208 dans les monastères de Cîteaux, La Ferté, Pontigny, Clairvaux et Morimond. Elle propose ainsi de dater cet acte de 1218, année qui coïnciderait davantage avec les abbés alors en fonction dans ces cinq monastères. Néanmoins, Sandrine Delaforge-Marchand date cet acte de 1208-1209 (DELAFORGE-MARCHAND Sandrine 1994, t. 1, p. 64-65, n 16)

${ }^{27}$ Archives nationales, LL 1595. Le cartulaire contient la copie de 137 actes concernant Paris et de nombreuses mentions d'autres actes (en tout, on peut recenser 280 documents).

${ }^{28}$ Bibliothèque nationale de France, ms. fr. 24432, f. $257 \mathrm{v}^{\circ} \mathrm{a}-261 \mathrm{v}^{\circ}$ b. MAREUSE Edgar (éd.) 1875. NICOLAS Catherine (éd.) 2012.

${ }^{29}$ Bove Boris 2004, p. 459, 463, 569 et 627.

${ }^{30}$ Pour la rive droite sont mentionnées les rues suivantes : «vicus sancti Pauli » (rue Saint-Paul, en dehors de la muraille) ; «Avenaria » (la Vannerie) ; «Mortelaria» (la Mortellerie) ; «vicus des Barres » (rue de la Barre) ; « vicus sancti Mederici » (rue Saint-Merry) ; «vicus qui dicitur la Texanderie » (la Tissanderie) ; «magnus vicus Parisiensis » (Grand-rue Saint-Denis) ; «vicus de Charoneria » (rue de la Charonnerie) ; «vicus de Tanneria » (rue de la Tannerie) ; «ruella Agnetis Bucherie »; «vicus Andrui Malet» (rue André Mallet); «vicus sancti Boniti» (rue Saint-Bon); «vicus Athacherie versus sanctum Bonitum» (rue de la Tacherie); «vicus Cuffariarum » (rues des Écouffes) ; « vicus de Marivaz » (rue de Marivaus) ; « vicus sancti Jacobi » (rue SaintJacques-la-Boucherie) ; « vicus qui dicitur vetus moneta » (rue de la vieille monnaie) ; «Sanneria » (la Saulnerie); «vicus Jardinorum» (la rue des Jardins) ; «Corrigiaria» (la Courroierie) ; «Faroneria » (la Ferronerie);
} 
parisien, comme la porte la porte Baudoyer («porta Bauderi »), la place de Grève («Gravia »), l'église Saint-Jacques-La-Boucherie, la porte Saint-Honoré, les planches Mibray («planche Mibrarii »), la maison du Château Fêtu («Chatel Fetu»), le marché des Champeaux («Campellis »), le Châtelet («Castelletum») ou l'église Saint-Leufroy («ecclesia sancti Leufredi »). Outre des mentions évoquant la manière dont ces rues sont appelées par les habitants (ce qui est le cas, par exemple, de la rue de la Tissanderie appelée « vicus qui dicitur la Texanderie »), une de ces rues reste sans nom et est simplement désignée comme : "vicus qui tendit a cuneo vici sancti Boniti versus Graviam ». Il pourrait s'agir de la « rue Jehan-PainMolet » évoquée par le Dit des rues de Paris $^{31}$. D'une manière générale, l'ordre de progression du cartulaire suit une logique de proximité géographique qui pourrait refléter l'organisation du chartrier $^{32}$.

Outre ces informations, un «rentier » daté de 1344 et ajouté à la suite du cartulaire $\left(\mathrm{ff} .87 \mathrm{r}^{\circ}-90 \mathrm{r}^{\circ}\right)$, se focalise sur les droits perçus dans 16 rues de la rive droite, en suivant un classement par infrastructures (ponts ou portes) ou par rues, ce qui donne une esquisse d'itinéraire, mais avec de nombreuses ruptures liées à l'éparpillement des possessions des moniales. L'énumération commence par le Pont Perrin, puis la rue de Jouy, la porte Baudoyer, la rue des Nonnains d'Yerres et la rue des Jardins, c'est-à-dire par l'espace parisien en relation directe avec le monastère de Saint-Antoine par la Grand rue de Saint-Antoine (qui n'y est pas mentionnée). Elle se poursuit avec la Vieille Tissanderie, la place de Grève, la Tannerie, les Commanderesses, la rue des Arcis, les deux Marivaux, pour passer par la Grand rue Saint-Denis dans les quartiers des Halles (La Truanderie, la porte Montmartre, la rue de la Croix du Tiroir, la Ferronnerie) et du Châtelet (la rue aux Lavandières, la Saulnerie, l'Écorcherie), avant de gagner l'île de la Cité et la rive gauche « oultre Petit Pont $»^{33}$.

Dans le cartulaire des années 1300, comme dans le « rentier » de 1344, on remarque que les cisterciennes ont une implantation en pointillé, tout simplement parce que contrairement aux monastères de Saint-Magloire ou de Saint-Martin, elles n'ont pas de véritable censive sur la rive droite ${ }^{34}$; elles possèdent en effet quelques maisons seulement par rue et des rentes. Des précisions apparaissent parfois sur la censive dans laquelle ces demeures se trouvent. Par exemple, pour le troisième acte copié dans le cartulaire et daté de juillet 1232, une courte analyse précise que la maison concernée se trouve dans le vicus Saint-Paul, dans la censive de

\footnotetext{
«Tonnelaria » (la Tonnellerie) ; «vicus Coconnerie» (rue de la Cossonerie); «Canaberia » (la Chanvrerie) ; «Truenderia » (la Truenderie).

${ }^{31}$ NiCOLAS Catherine (éd.) 2012, p. 92.

${ }^{32}$ L'ordre dans lequel sont transcrits les actes de la première partie du cartulaire de Saint-Antoine-des-Champs (Archives nationales, LL 1595, f. $5 \mathrm{r}^{\circ}-58 \mathrm{r}^{\circ}$ ) correspond sans doute à l'ordre dans lequel sont conservées les chartes originales. L'organisation du chartrier est en effet visible dans ce recueil à travers des indications comme : «posite in primo, secundo, tercio, quarto ergastulo primi raustri (loci superioris a parte ecclesie) », puis «in primo, secundo, tertio, quarto ergastulo secundi raustri (loci superioris) » et enfin « in primo ergastulo tercii raustri ». POULLE Emmanuel 1996, p. 347.

${ }^{33}$ Les termes utilisés dans le rentier pour désigner les rues sont les suivants : « au pont Perrin »; « en la rue de Joy »; « a la porte Baudoer »; « en la rue aus nonains d'Yerre »; « au viel cimetiere Saint-Jehan »; « en la rue des Jardins »; « en la vielz Tisseranderie » ; « en Greve »; « en la Tennerie »; « aus Commanderesses »; « en la rue des Arssis »; « en Mariva[u]x »; « en petit Marivaux »; « en la grant rue Saint-Denis »; « aus halles de Paris »; « en la Truanderie »; « a la porte de Montmartre»; «en la rue de la crois du Tiroer»; «en la Ferronnerie »; «pres du Chastelet»; « en la rue des Lavandieres»; « en la Saunerie»; «devant SaintHeuffroy »; « en l'Eschorcerie »; « en la Cité »; « oultre-petit-pont ».

${ }^{34}$ WEISS Valentine 2009, t. I, p. 234.
} 
Saint-Eloi ${ }^{35}$, dont le prieur apparaît comme le donateur d'une rente en $1281^{36}$. D'une manière générale, on doit souligner la forte capacité d'adaptation des cisterciennes aux réalités de la ville. Néanmoins, ce cartulaire, comme les censiers qui lui sont associés, montre qu'elles ne participent pas directement à l'urbanisation de la rive droite de Paris. Elles ne font qu'accepter des maisons et les cens qui pèsent sur elles et ne construisent pas un espace qui leur serait propre.

Saint-Magloire et Saint-Martin-des-Champs sont deux établissements anciens, bien implantés à Paris et dans ses environs au XIII ${ }^{\mathrm{e}}$ siècle, qui ont mené pendant la période précédente une politique de lotissement dans leur censive respective. Saint-Antoine-des-Champs est un monastère nouveau installé en marge de l'espace parisien, dont l'influence sur le réseau viaire a été des plus réduites. Néanmoins, leurs livres d'archives reflètent des pratiques communes, à savoir l'adoption d'une organisation s'appuyant sur les rues de Paris. En revanche, ils ne montrent que d'une manière marginale la structuration progressive des espaces ruraux situés à l'extérieur des murailles de Paris.

\section{Des livres d'archives comme reflet de la structuration progressive en rues des espaces marginaux à la fin du XIII ${ }^{\mathrm{e}}$ siècle}

Les livres d'archives de Saint-Eloi et du Temple permettent de mettre en lumière la conquête progressive de la ville sur les espaces encore ruraux. Elle se fait à travers le percement de nouvelles rues qui reprennent parfois le tracé d'anciens chemins vicinaux ou sont créées ex nihilo.

Pour Saint-Eloi, prieuré dépendant de Saint-Maur des Fossés et situé dans l'île de la Cité, nous avons un document exceptionnel de précision sur la censive du monastère, qui s'étend à l'intérieur des murailles sur le Bourg Thibourg et, extra-muros, dans le quartier Saint-Paul. Le censier daté de 1391 transmet en fait un état des lieux de la fin du XIII ${ }^{\mathrm{e}}$ siècle $^{37}$. Au début de ce livre d'archives (aux ff.6r-9r), avant le censier à proprement parler, se trouvent deux textes utiles pour mener une étude topographique de l'est parisien : une longue charte en latin de Philippe III le Hardi (1270-1285) datée d'août $1280^{38}$, précédée par un texte en français qui pourrait être sa traduction ou un document préparatoire ${ }^{39}$. Le titre de ce dernier est explicite et permet de comprendre les enjeux de leur copie à la tête du censier : «Cest l'ordenance au prieur de S. Eloy de Paris comment la terre de Saint Pol et d'ailleurs de Paris doit etre ebournee ». Comme le mot ebourner veut dire borner, il est aisé de saisir que ces deux textes sont les témoins de la délimitation de la censive, suite à un conflit entre les agents royaux et le prieur.

La censive du prieuré est en fait un espace en expansion démographique et économique, principalement à l'extérieur de la muraille de Philippe-Auguste ${ }^{40}$, dans la paroisse Saint-Paul, objet de toutes les convoitises à cause de son dynamisme et de ses potentialités. Outre le rempart $\mathrm{du} \mathrm{XIII}{ }^{\mathrm{e}}$ siècle dès lors débordé par cet essor, y est également inclus l'ancien mur du XI $\mathrm{I}^{\mathrm{e}}$ siècle

\footnotetext{
${ }^{35}$ Archives nationales, LL 1595, f. 6r $\mathrm{r}^{\circ}, \mathrm{n}^{\circ} 3$ : « Nota alia littera de viginti quinque solidis qui debent recipi in domo Roberti le Tort in vico Sancti Pauli et in censiva Sancti Eligii qui sic incipit : Omnibus presentes litteras inspecturis, officialis curie Parisiensis salutem in Domino. Et sic terminatur : Actum anno domini millesimo ducentesimo trecentesimo secondo mense Julio ».

${ }^{36}$ Archives nationales, LL 1595, ff. $5 \mathrm{v}^{\circ}-6 \mathrm{r}^{\circ}, \mathrm{n}^{\circ} 2$.

${ }^{37}$ SUMPF Alexandre 1998-1999, p. 9.

${ }^{38}$ Archives nationales, LL 75, ff. $7 \mathrm{r}^{\circ}-9 \mathrm{r}^{\circ}$. Le titre du diplôme royal, écrit en textualis libraria, est des plus simples : «Sequitur carta super ordinacione predicta » et semble indiquer que cet acte serait considéré comme un soutien ou une preuve du texte écrit en français. Voir SUMPF Alexandre, 1988-1999, Annexes, Pièce annexe n 2, p. 4-8. GUERARD Benjamin 1850, t. 3, p. 277-281, n 8. L'original en est conservé : Archives nationales, S 1182 n $^{\circ} 20$.

${ }^{39}$ Archives nationales, LL 75, ff. $6 \mathrm{r}^{\circ}-7 \mathrm{r}^{\circ}$.

${ }^{40}$ La mention des hôtes (hospites) dans le diplôme royal est un indice de cette expansion démographique dans la partie rurale de la censive.
} 
qui ne sert plus que d'élément de repère : la charte en latin évoque en effet les «muri veteres Parisienses », et l'ordonnance en français, les «viez murs de Paris ». Grâce à ces deux documents, sont repérables dix-sept rues ou parties de rues réparties autour de l'axe de la rue Saint-Antoine (Grand-rue de la Porte Baudoyer et rue du Pont-Perrin). Les deux documents étudiés permettent bien de cerner les deux pôles de la censive et de supposer leur complémentarité entre un espace déjà urbain (le Bourg Thibourg) et un espace encore pionnier autour de Saint-Paul ${ }^{41}$, qui attira au XIV ${ }^{\mathrm{e}}$ siècle une résidence royale ${ }^{42}$. Dans le quartier SaintPaul néanmoins, d'après la documentation disponible, à savoir le chartrier ${ }^{43}$ et le censier, il n'y a pas de traces d'intervention directe de Saint-Eloi dans le tracé des rues ou dans sa mise en lots, qui aurait plutôt été le fait de particuliers : Alexandre Sumpf souligne en effet la présence de grandes propriétés avoisinant de simples camere, c'est-à-dire des chambres ou des pièces localisées principalement dans la rue des Jardins, avec la persistance d'exploitations agricoles ${ }^{44}$. Il semblerait en outre que des chemins aient existé préalablement au percement des rues, ne serait-ce que pour aller à l'église (rue Saint-Paul) ou pour circuler le long du fossé au pied du rempart (la rue des Jardins).

Si les moines de Saint-Eloi ont eu, somme toute, une politique peu interventionniste sur le réseau viaire de leur censive, les Templiers en revanche ont été bien plus actifs à la fin du XIII $^{\mathrm{e}}$ siècle. D'après les travaux de Geneviève Étienne, trois ensembles de bâtiments localisés à des emplacements différents ont été édifiés par les Templiers dans Paris : un premier ensemble localisé au chevet de l'église Saint-Jean en Grève considéré comme le Vieux Temple, un deuxième dans la couture appelé Novum Templum en 1274 et un dernier qui serait situé au chevet de l'église Saint-Gervais ${ }^{45}$. À partir de ces trois espaces, les Templiers ont participé à l'urbanisation parisienne en pratiquant une politique de lotissement dans le quartier du Marais actuel, particulièrement visible dans la Villeneuve du Temple extra-muros ${ }^{46}$. Cet interventionnisme peut être étudié à travers la copie moderne du premier censier élaboré entre 1227 et $1235^{47}$, d'un censier de $1252-1253^{48}$, d'un acte du roi Philippe le Hardi daté d'août $1279^{49}$, et enfin d'un registre de saisines couvrant la période $1287-1307^{50}$.

L'acte royal de 1279 est la notification d'un compromis conclu entre le prévôt de Paris et les Templiers pour mettre fin à une querelle à propos des droits de justice que les religieux prétendaient avoir sur toutes leurs possessions à l'intérieur et à l'extérieur de la muraille et que le prévôt leur contestait. Pour ce faire, il donne les limites des droits et des possessions du Temple. Pour la terre du Temple extra-muros notamment, le processus de délimitation se fait avec une grande précision à cause de l'importance de l'enjeu pour les deux parties en présence :

«... a porta seu posterna que vulgariter dicitur posterna Barbete secundum quod dicti muri se protendunt ab illa posterna usque ad portam vici quid dicitur vicus Templi ${ }^{51}$; item ab eadem porta vici Templi sicut vicus seu strata protenditur usque ad fossatum dicitur vulgariter le fossé la Boucelle quod fossatum ab ipsa strata inter saliceta domus Templi et terram Unfredi Nufle intermedium consistit; item ab illo loco et eadem strata ac dicta

\footnotetext{
${ }^{41}$ SUMPF Alexandre 1998-1999, p. 124.

${ }^{42}$ GUERBER Estelle 2001.

${ }^{43}$ Archives nationales, S 1182-1186B.

${ }^{44}$ SUMPF Alexandre 1998-1999, p. 104 et sq. ; p. 108 et sq.

${ }^{45}$ ETIENNE Geneviève 1974, p. 5-17.

46 ThOMAs Nicolas, Bourlet Caroline, Roms Cédric 2007 p. 60-67.

${ }^{47}$ Archives nationales, $\mathrm{R}^{3} 10$.

${ }^{48}$ Archives nationales, MM 128 (anc. M 75, musée 255).

${ }^{49}$ Archives nationales, K $34 \mathrm{n}^{\circ} 24$.

${ }^{50}$ Archives nationales, S 5594.

${ }^{51}$ Il semblerait que l'expression « vicus Templi » puisse être traduite ici par «bourg du Temple » et non « rue du Temple ».
} 
terra Unfredi Nufle veluti fossatum se comportat usque ad cuneum cortillie Barbete a parte camporum et ab inde et chemino scilicet quod communiter vocatur cheminum Menilii Mantens, sicut idem cheminum ducit de loco predicto Parisius ad posternam Barbete supradictam ».

Ainsi, dans cet espace situé entre, «au nord-est, l'actuelle rue de la Folie-Méricourt, à l'est les actuelles rues Oberkampf, des Filles-du-Calvaire et Vieille-du-Temple, au sud l'enceinte, à l'ouest et au nord-ouest, les actuelles rues du Temple et du Faubourg-du-Temple et l'ancien fossé La Boucelle $»^{52}$, les Templiers obtiennent la confirmation du droit de haute et de basse justice qu'ils perdent, par le même acte, dans l'espace intra-muros au profit du roi et du prévôt de Paris. En outre, pour attirer des habitants (appelés hospites) dans la couture, des privilèges confirmés par le roi (exemption de taille, d'ost, de chevauchée, de guet, de tonlieu et de coutume) leur sont offerts, même si l'exemption de la taille n'a pas été vraiment respectée par les agents royaux ultérieurement ${ }^{53}$. Néanmoins, grâce à ces privilèges, la Villeneuve du Temple s'est rapidement urbanisée entre 1279 et $1307^{54}$. Dans ce secteur en effet, se met en place un véritable lotissement dont témoigne la régularité du tracé de la voirie avec des rues parallèles ou perpendiculaires. À partir de 1282, les Templiers créent, entre l'enceinte de Paris au sud et leur enclos au nord, neuf nouvelles rues ${ }^{55}$.

En 1288, pour faciliter leur entreprise, ils obtiennent du roi le droit d'ouvrir une nouvelle porte dans la muraille : il s'agit de la porte du Chaume. À partir de là, la couture du Temple se structure autour d'un axe médian existant dès 1288 et partant de la Porte du Chaume vers le Temple : il a d'abord été appelé rue Neuve du Temple, puis rue de la Nouvelle Poterne, rue de la Porte Neuve et enfin rue du Chaume (l'actuelle rue des Archives). De part et d'autre de cet axe, on trouve les rues du Temple et la rue Barbette dans la prolongation de la rue Vieille du Temple, sachant que la rue Barbette ne prend ce nom qu'au XIV e siècle. Selon Caroline Bourlet et Nicolas Thomas, deux phases de construction sont repérables à partir des documents étudiés ${ }^{56}$. Ainsi, depuis la rue du Chaume, ont été ouvertes trois rues : la rue de Paradis (1288); la rue du Chantier (1293) ; la rue des Quatre fils Hémon (l'actuelle rue des Quatre-Fils) citée à partir de 1305. De la rue du Temple, partent cinq autres rues : la rue des Étuves, citée dès 1296 et devenue la rue des Bouchers après 1303 ; la rue Jean L'Huillier, citée depuis 1287 ; la rue des Noyers ou du Noyer (depuis 1297) ; la rue Jean de Saint-Quentin (1296), devenue rue Grognet en 1296-1297, puis rue Pastourelle (1299) ; la rue Richard des Poulies (1296). Ces rues peuvent être classées en trois ensembles : les premières portent des noms liés au Temple lui-même, comme la rue Vieille-du-Temple ou la rue Neuve-du-Temple avant son changement de nom ; les deuxièmes ont des dénominations liées à des bâtiments spécifiques, à des activités économiques ou agricoles, comme la rue des Étuves ou la rue du Chantier ; enfin, les dernières sont appelées d'après des personnes qui y sont possessionnées, comme la rue Jean l'Huillier ou la rue Jean de Saint-Quentin.

Si à la fin du XIII ${ }^{\mathrm{e}}$ siècle, les actes comme les livres d'archives des établissements sélectionnés montrent de la part des religieux une attitude parfois différente face au réseau viaire parisien et à sa structuration progressive dans les espaces encore ruraux, en revanche, ils permettent de mettre en évidence une stabilisation de rues par le passage à l'écrit des noms entre les années 1260 et 1310.

\section{La stabilisation des noms de rues par le passage à l'écrit}

\footnotetext{
52 ETIENNE Geneviève 1975 p. 88. Voir également : EAD. 1974, p. 203.

${ }^{53}$ EAD. 1974, p. 206.

${ }^{54}$ EAD. 1975 , p. 87.

${ }^{55}$ HAMON Etienne 2012, p. 50 et 52. ETIENNE Geneviève 1975, p. 91-92.

56 Thomas Nicolas, Bourlet Caroline, Roms Cédric 2007, p. 66.
} 
Si les écrits, par leur organisation, sont un reflet du réseau viaire parisien et de sa structuration extra-muros, ils ont également un rôle important dans la stabilisation des noms de rues à la fin du XIII ${ }^{\mathrm{e}}$ siècle. Le passage à l'écrit permet en effet de figer pour un temps la toponymie et de transmettre un état des lieux à un moment donné.

Une première remarque doit être faite avant d'étudier cette fixation par l'écrit des toponymes. Il s'agit de la différenciation dans la documentation de trois types d'espaces selon leur dénomination : des rues appelées vicus principalement intra-muros ; ce terme semble faire référence à des rues déjà structurées, parfois depuis longtemps ; des rues non précédées du terme vicus, principalement dans le bourg l'Abbé pour Saint-Magloire (Le Huleu, Darnestat), ce qui ferait référence à un état intermédiaire, mais des rues déjà bien structurées et situées dans Paris (comme la Mortellerie, la Tannerie, la Vieille Tissanderie, etc.) ne sont pas précédées par ce terme et cette absence laisse supposer qu'il s'agit plutôt d'espaces « fonctionnels » désignés par les activités anciennes ou récentes qui s'y sont implantées ${ }^{57}$; un espace enfin qui ne contient pas de mention de vicus ou même de nom pouvant laisser croire en l'existence d'une rue et qui correspond à un espace encore agricole.

À partir de cette rapide mise au point conceptuelle, pour étudier ce qui nous importe ici, à savoir la stabilisation des toponymes par l'écrit, trois exemples peuvent être utilisés, à savoir les censiers du Temple, de Saint-Martin-des-Champs et de Saint-Eloi. Dans le premier cas, les rues tracées à l'initiative des Templiers, nous l'avons vu précédemment, changent de noms entre 1280 et 1300 . Ces derniers semblent ensuite se stabiliser entre 1300 et 1307, date de l'interruption du lotissement liée à l'arrestation des Templiers. D'une manière générale, ils ne semblent pas avoir été donnés par les religieux eux-mêmes, mais plus probablement par leurs habitants-usagers. L'action templière aurait donc seulement porté sur le percement des voies nouvelles, mais pas sur la fixation des toponymes. Ainsi, leurs documents livrent l'état de la toponymie au nord-est de Paris avant 1307.

Plus intéressant pour notre propos est l'exemple de Saint-Martin-des-Champs. En comparant les quatre censiers des années 1263-1300, on repère une vingtaine de noms de rues qui ne sont pas agencés de la même façon selon les termes. En 1263, par exemple, l'énumération commence pour les quatre termes par les «vicus majoris et vicus de Fripelon », mais change par la suite en fonction des censitaires taxés à ces quatre moments de l'année. La même constatation vaut pour l'année 1274, qui débute toutefois par les cens perçus «in magno vico Sancti Martini per extra muros », tout comme en 1293 et en 1300. Cette insistance sur la grande rue, qui se retrouve également dans les manuscrits de Saint-Magloire (pour la rue Saint-Denis notamment) montre l'importance que peut avoir une rue plus large que les autres à la fois dans la topographie, dans la structuration du réseau viaire et dans les conceptions spatiales des habitants.

Tableau 1 : La stabilisation des noms de rues à travers les censiers de Saint-Martin-des-Champs entre 1263 et 1300

\begin{tabular}{|l|l|l|l|}
\hline Censier de 1263 & Censier de 1274 & Censier de 1293 & Censier de 1300 \\
\hline
\end{tabular}

\footnotetext{
${ }^{57}$ Caroline Bourlet a proposé cette hypothèse lors de son étude des itinéraires de percepteurs dans les rôles de taille (https://irht.hypotheses.org/1691). Ainsi, elle remarque qu' " en 1296-1300, certains espaces ne sont jamais appelés rues. Ils se trouvent essentiellement sur la rive droite ou sur la Cité, et portent le nom d'une activité, comme par exemple la Pelleterie (Cité), la Tannerie, la Heaumerie etc. À l'exception, peut-être, de deux d'entre eux dont le nom se rapporte à autre chose qu'une activité (les fossés St-Germain qui sont d'anciens fossés et la Bretonnerie qui est le quartier où sont implantés les Bretons dès le début du XIII ${ }^{\mathrm{e}}$ siècle), ces espaces peuvent être qualifiés de «fonctionnels » pourvus, au moins dans l'imaginaire bourgeois, d'une forte personnalité et perçus comme des espaces, peu assimilables à une voie ou une partie de voie parce qu'ils en déborderaient les limites. Ils portent souvent le témoignage d'espaces fonctionnels antérieurs, qui ont plus ou moins perduré. Ces espaces sont si fortement identifiés et connus pour conserver leur dénomination jusqu'au début du XIV siècle au moins, et pour servir fréquemment de repère lors de la description des itinéraires des rues proches ».
} 


\begin{tabular}{|c|c|c|c|}
\hline $\begin{array}{l}\text { In vico Majoris et in } \\
\text { vico qui dicitur } \\
\text { Fripelon }\end{array}$ & $\begin{array}{l}\text { Vicus Majoris Sancti } \\
\text { Martini de Campis }\end{array}$ & $\begin{array}{l}\text { Vicus Majoris Sancti } \\
\text { Martini }\end{array}$ & $\begin{array}{l}\text { Vicus Majoris Sancti } \\
\text { Martini }\end{array}$ \\
\hline $\begin{array}{l}\text { Vicus Templi qui ducit } \\
\text { a puteao de Fripelon } \\
\text { usque ad calceyam } \\
\text { Templi et de super } \\
\text { calceaym usque ad } \\
\text { muros Parisius }\end{array}$ & $\begin{array}{l}\text { Vicus qui ducit a puteo } \\
\text { de Ferpellione usque } \\
\text { ad muros Parisienses in } \\
\text { vico Templi }\end{array}$ & Vicus Ferpilonis & $\begin{array}{l}\text { Vicus Ferpilonis et } \\
\text { vicus Templi }\end{array}$ \\
\hline Vicus Roberti Begonis & Roberti & Vicus Roberti Begonis & Vicus Roberti Begonis \\
\hline Vicus Guerini Boucel & Vicus Guerini Boucel & Vicus Guerini Boucelli & Vicus Guerini Boucelli \\
\hline $\begin{array}{ll}\text { Vicus } & \text { Geleberti } \\
\text { Gravelarii } & \\
\end{array}$ & $\begin{array}{l}\text { Vicus Gravelarii sive } \\
\text { vicus pavatus }\end{array}$ & Vicus Gravalarii & Vicus Gravelarii \\
\hline $\begin{array}{l}\text { Vicus Cimiterii Sancti } \\
\text { Nicholai }\end{array}$ & $\begin{array}{l}\text { Vicus Cimiterii Sancti } \\
\text { Nicholai de Campis } \\
\text { Parisiensis }\end{array}$ & $\begin{array}{l}\text { Vicus Cymiterii Sancti } \\
\text { Nicholai }\end{array}$ & $\begin{array}{l}\text { Vicus Cymiterii Sancti } \\
\text { Nicholai }\end{array}$ \\
\hline $\begin{array}{l}\text { Vicus Garneri de } \\
\text { Sancto Lazaro et juxta } \\
\text { posternam Nicholai } \\
\text { Hydolon }\end{array}$ & $\begin{array}{lll}\text { Vicus Garneri } & \text { de } \\
\text { Sancto Lazaro } & \end{array}$ & $\begin{array}{l}\text { Vicus Guerneri de } \\
\text { Sancto Lazaro }\end{array}$ & $\begin{array}{l}\text { Vicus Garnerii } \mathrm{de} \\
\text { Sancto Lazaro }\end{array}$ \\
\hline $\begin{array}{llr}\text { Vicus } & \text { Sancti } & \text { Martini } \\
\text { per extra } & \text { muros } \\
\text { Parisienses } & \\
\end{array}$ & $\begin{array}{l}\text { In magno vico Sancti } \\
\text { Martini de Campis per } \\
\text { extra muros }\end{array}$ & $\begin{array}{l}\text { In magno vico Sancti } \\
\begin{array}{l}\text { Martini per extra } \\
\text { muros }\end{array}\end{array}$ & $\begin{array}{l}\text { Magnus vicus Sancti } \\
\text { Martini de Campis per } \\
\text { extra muros }\end{array}$ \\
\hline Vicus Joculatorum & Vicus Joculatorum & Vicus Joculatroum & Vicus Joculatorum \\
\hline Vicus Plastrarie & Vicus Plastrarie & Vicus Plastrarie & Vicus Plastrarie \\
\hline $\begin{array}{l}\text { Vicus Godefridi de } \\
\text { Balneolis }\end{array}$ & $\begin{array}{lr}\text { Vicus Godefridi de } \\
\text { Balnelois } & \text { sive } \\
\text { Stuparum } & \\
\end{array}$ & $\begin{array}{l}\text { Vicus Godefridi de } \\
\text { Balneolis }\end{array}$ & $\begin{array}{lr}\text { Vicus Gaufridi } & \text { de } \\
\text { Balneolis } & \text { sive } \\
\text { Stupharum } & \\
\end{array}$ \\
\hline $\begin{array}{l}\text { Vicus Belli Burgi / qui } \\
\text { dicitur Pulcher Burgus }\end{array}$ & Vicus Belli Burgi & Vicus Belli Burgi & Vicus Belli Burgi \\
\hline $\begin{array}{ll}\text { Vicus } & \text { Symonis } \\
\text { Franque } & \\
\end{array}$ & & $\begin{array}{ll}\text { Vicus } & \text { Symonis } \\
\text { Franque } & \\
\end{array}$ & $\begin{array}{ll}\text { Vicus } & \text { Symonis } \\
\text { Franque } & \\
\end{array}$ \\
\hline Vicus de $\mathrm{Cu}$ de $\mathrm{Sac}$ & Vicus de Cul de Sac & Vicus de Cul de Sac & Vicus de Cul de Sac \\
\hline Vicus Sine Capite & $\begin{array}{l}\text { Vicus Sine Capite utra } \\
\text { crucem Regine }\end{array}$ & $\begin{array}{l}\text { Vicus Sine Capite ultra } \\
\text { crucem Regine }\end{array}$ & $\begin{array}{l}\text { Vicus Sine Capite ultra } \\
\text { crucem Regine }\end{array}$ \\
\hline \multicolumn{4}{|l|}{ Vicus de Quiquenpoist } \\
\hline 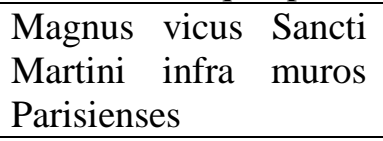 & $\begin{array}{l}\text { Magnus vicus Sancti } \\
\text { Martini per infra muros }\end{array}$ & $\begin{array}{l}\text { Magnus vicus per infra } \\
\text { muros }\end{array}$ & $\begin{array}{l}\text { Vicus Sancti Martin de } \\
\text { Campis per infra muros }\end{array}$ \\
\hline In Gravia & In Gravia & & \\
\hline \multirow[t]{6}{*}{$\begin{array}{l}\text { Magnus vicus sancti } \\
\text { Dyonisii }\end{array}$} & $\begin{array}{l}\text { Magnus vicus Sancti } \\
\text { Dyonisii Parisiensis }\end{array}$ & $\begin{array}{l}\text { Vicus Sancti Dyonisii } \\
\text { per infra muros }\end{array}$ & $\begin{array}{l}\text { Vicus Sancti Dyonisii } \\
\text { per infra muros }\end{array}$ \\
\hline & $\begin{array}{l}\text { Vicus ubi coquuntur } \\
\text { anseres juxta vicum de } \\
\text { Quiquenpoit }\end{array}$ & & $\begin{array}{l}\text { Vicus ubi coquuntur } \\
\text { anseres }\end{array}$ \\
\hline & $\begin{array}{l}\text { Vicus Michaelis dicti } \\
\text { Comitis }\end{array}$ & $\begin{array}{ll}\text { Vicus } & \text { Michaelis } \\
\text { Comitis } & \end{array}$ & $\begin{array}{ll}\text { Vicus } & \text { Michaelis } \\
\text { Comitis }\end{array}$ \\
\hline & $\begin{array}{l}\text { In Carnificeria } \\
\text { Parisiensis }\end{array}$ & $\begin{array}{l}\text { In Carneficeria } \\
\text { Parisiensis }\end{array}$ & $\begin{array}{l}\text { In Carnificeria } \\
\text { Parisiensis }\end{array}$ \\
\hline & $\begin{array}{l}\text { In Vaneria / in } \\
\text { Aveneria }\end{array}$ & & In Avenaria \\
\hline & Vicus Mali Consilii & & \\
\hline
\end{tabular}




\begin{tabular}{|l|l|l|l|}
\hline & & $\begin{array}{l}\text { Vicus Alberici } \\
\text { Carnificis }\end{array}$ & $\begin{array}{l}\text { Vicus Alberici } \\
\text { Carnificis }\end{array}$ \\
\hline & & Vicus de Correaria & Vicus de Correarii \\
\hline & & $\begin{array}{l}\text { Vicus de Trousse } \\
\text { Vache }\end{array}$ & Vicus de Troussevache \\
\hline & $\begin{array}{l}\text { Ruella Amaurici de } \\
\text { Royssi }\end{array}$ & Vicus Emarii de Royssi \\
\hline
\end{tabular}

Ces quatre censiers reprennent l'un après l'autre les mêmes toponymes, mises à part des variantes orthographiques comme pour la rue Frépillon (actuelle rue Volta), orthographiée Fripelon, Frepelion ou encore Ferpellion. Ainsi, la toponymie semble stable dans la censive de Saint-Martin dans la deuxième moitié du XIII siècle. Si ces toponymes subissent peu de modifications, des précisions peuvent néanmoins être ajoutées, notamment pour le «vicus Gileberti/Geleberti Gravelarii » de 1263 qui est dit «vicus Gravelarii sive vicus pavatus » en 1274 , puis de nouveau « vicus Gravalarii » en 1293 et « vicus Gravelarii » en $1300^{58}$; le « vicus Godefridi de Balneolis » de 1263 est mentionné comme «vicus Godefridi de Balneolis sive Stuparum » en 1274, pour mettre en évidence les activités qui y sont localisées, «vicus Godefrede de Balneolis » en 1293 et « vicus Gaufridi de Balneolis sive Stupharum » en 1300. Ce nom de «rue des Etuves » est finalement resté au détriment de la première dénomination. Dans le censier de 1274, la rue aux Oies, désignation qui se retrouve dans d'autres documents depuis le début du XIII ${ }^{\mathrm{e}}$ siècle $^{59}$, est identifiée par une périphrase qui met en évidence sa fonction première : «vicus ubi coquuntur anseres ». . Dans ce cas néanmoins, le copiste indique en plus qu'elle se trouve à côté de la rue Quincampoix, précision qui disparaît dès le quatrième terme du censier de 1274 et qui est absente des deux suivants, ce qui peut vouloir dire qu'il s'agissait de nouveaux cens à percevoir et qu'il était alors nécessaire de les localiser précisément à partir d'une rue déjà citée en 1263, démarche qui ne semble plus utile au scribe par la suite.

Pour Saint-Eloi enfin, la comparaison entre les termes latins et français de l'acte royal et de l'ordonnance montre soit l'utilisation de termes vernaculaires dans le texte latin, comme le pont Perrun par exemple, soit leur latinisation pure et simple. Le terme vicus utilisé pour désigner les rues dans le diplôme de Philippe III est traduit par rue dans le texte français. Ce phénomène est plus visible encore dans le cas de la rue Pierciee qui est désignée ainsi, aussi bien dans le document en français que dans celui en latin.

Tableau 2 : Comparaison des termes latins et français utilisés dans l'ordonnance en français et dans le diplôme en latin dans le censier de Saint-Eloi

\begin{tabular}{|c|c|}
\hline $\begin{array}{l}\text { Censier de Saint-Eloi, Archives nationales, LL } \\
75 \text {, Ordonnance en français (ff. 6r-7r) }\end{array}$ & $\begin{array}{l}\text { Censier de Saint-Eloi, Archives nationales, } \\
\text { LL 75, Ordonnance en latin (ff. 7r-9r) }\end{array}$ \\
\hline porte Baudoier & porta Bauderii \\
\hline meson a la Guespine ${ }^{60}$ & domusa la Guespine \\
\hline meson de Joi61 & domus de Joiaco \\
\hline rue Saint-Anthoine & magnus vicus Sancti Anthonii \\
\hline rue Pierciee & rue Pierciee \\
\hline rue de la Posterne Saint Pol & vicus posterne Sancti Pauli \\
\hline Saint Pol & Sanctus Paulus \\
\hline Les Barrés ${ }^{62}$ & Domus fratrum Beate Marie de Carmello \\
\hline
\end{tabular}

\footnotetext{
${ }^{58}$ C'est la rue aux Graveliers.

${ }^{59}$ HiLlairet Jacques 1963, t. 2, p. 207.

${ }^{60}$ La ruelle a la Guespine est appelée ainsi d'après la « meson a la Guespine » qui est mentionnée aussi bien dans l'ordonnance en français que dans le diplôme en latin.

${ }^{61}$ La rue de Jouy tire son nom de la maison des moines cisterciens de Jouy-en-Brie (Chenoise, Seine-et-Marne).

${ }^{62}$ Les Carmes (ou Barrés) sont installés dans le quartier Saint-Paul depuis 1256.
} 


\begin{tabular}{|l|l|}
\hline porte de Barbeel & porta murorum de Barbeel \\
\hline jusques a Saine & vicus per quem itur inferius versus Secanam \\
\hline Pont Perrun & Pons Perrun \\
\hline rue Moitoiene & vicus Medietarius / Medietaneus \\
\hline chauciée de la rue Saint Anthoine & Calceia vici sancti Anthonii \\
\hline chauciée du Pont Perrin & Calceya de ponte Perrini \\
\hline ruele au roy de Sezile & vicus regis Sicilie \\
\hline rue Anquetin le Faucheur & vicus Anquetini Falcatoris \\
\hline Viez rue du Temple & vicus veteris Templi \\
\hline La Bretonnerie & La Bretonneria \\
\hline Bourg Thibot & Burgus Thiboudi \\
\hline place du Viez semetire Saint Jean & platea Veteris cimiterii Sancti Johannis \\
\hline rue du Franc Murier & ruella dou Franc Mourier \\
\hline
\end{tabular}

À travers ces deux documents, apparaît en fait un état des rues et de leurs noms vers 1280 . Dans le Bourg Thibourg, les noms comme le tracé des rues semblent déjà stabilisés à cette époque. En revanche, dans le quartier Saint-Paul, des modifications toponymiques sont intervenues rapidement, comme pour la rue de la [fausse] poterne Saint-Paul ${ }^{63}$, prolongation de la rue de Jouy. Dans le rôle de la taille de 1300, elle est dénommée « rue des Poulies » et, dans le Dit des rues de Paris, « rue des Poulies-Saint-Pou ${ }^{64}$. Par la suite, elle devient rue des Prêtres Saint-Paul ou rue de l'archet Saint-Paul ${ }^{65}$. La rue Moitoiene est appelée « rue des Jardins » dans le censier ${ }^{66}$, nom qu'elle conserve dans le rôle de taille de 1300 . La grande rue qui prolonge la rue de la porte Baudoyer est nommée, à la fois dans l'ordonnance et dans le diplôme, " grande rue Saint-Antoine », d'après le nom du monastère de moniales cisterciennes, preuve de la prise en compte de leur présence par les Parisiens et par l'autorité royale, alors que cette dénomination est absente de leur cartulaire. Enfin, certaines rues en cours de structuration sont encore sans nom fixe à la fin du XIII ${ }^{\mathrm{e}}$ siècle comme le «vicus per quem itur inferius versus Secanam ».

À travers les livres d'archives, se dessine l'urbanisation progressive des espaces convoités à l'intérieur et à l'extérieur des remparts, à partir de véritables fronts pionniers. Au fur et à mesure que la ville s'étend, des noms de rues apparaissent dans les différents recueils. Ce sont ceux qui sont utilisés couramment par les habitants et non des toponymes imposés par les religieux. En les écrivant dans leurs livres d'archives, ces derniers ont la volonté de se conformer aux usages parisiens pour éviter les contestations, mais, ce faisant, ils participent de leur stabilisation et livrent les contours de leur carte mentale de la capitale et de ses faubourgs.

\section{Conclusion}

La structuration de l'espace parisien en rues au cours du XIII ${ }^{\mathrm{e}}$ siècle est perceptible à travers les documents d'archives qui permettent de distinguer deux espaces : d'abord un espace fortement bâti et densément peuplé, majoritairement situé à l'intérieur de la muraille de Philippe-Auguste dont les rues sont souvent appelées vicus; ensuite un espace en cours d'urbanisation, dans lequel plusieurs usages sont en concurrence (notamment les usages agricoles ou récréatifs), à cheval sur la muraille. À travers la documentation, se devine ainsi la progression de la ville, qui s'accompagne d'une apparition plus ou moins rapide des noms de rues $^{67}$. Ces derniers se fixent dans les années 1270-1310 et leur mise par écrit, dans les livres

\footnotetext{
${ }^{63}$ La dénomination de « vicus false posterne » apparaît dans le censier au f. 23 v .

${ }^{64}$ NiCOLAS Catherine (éd.) 2012, p. 104-105.

${ }^{65}$ SUMPF Alexandre 1998-1999, p. 32.

${ }^{66}$ Ibid., p. 26. Archives nationales, LL 75, f. $11 \mathrm{r}^{\circ}$ : « Tunc sequntur census vici de Jardinis qui solebat vocari vicus Medietaneus »; f. $54 \mathrm{r}^{\circ}$ : «Et eundum est ad vicum de Jardinis que solebat dici Medietaneus ».

${ }^{67}$ LEGUAY Jean-Pierre 1984.
} 
d'archives comme dans les textes narratifs, participe de leur stabilisation au début du XIV siècle.

Marlène Helias-Baron

Ingénieure de recherche

IRHT-CNRS

40 avenue d'Iéna

75116 Paris 


\section{Bibliographie}

Bertrand Paul, 2015, Les écritures ordinaires. Sociologie d'un temps de révolution documentaire (1250-1350), Paris, Publications de la Sorbonne

BOURLET Caroline et PoISSON Jean-François, 2016, « Itinéraire des percepteurs et marquage de l'espace urbain (1299-1300) », dans Séminaire d'histoire de Paris [6] du 18 mars 2016, voir : https://irht.hypotheses.org/1691

Bove Boris, 2004, Dominer la ville. Prévôts des marchands et échevins parisiens de 1260 à 1350, Paris, Editions du CTHS

DELAFORGE-MARCHAND Sandrine, 1994, Édition du chartrier de l'abbaye de Saint-Antoinedes-Champs (1191-1256), 3 vol., Thèse de l'École nationale des chartes

DESCIMON Robert et WeISS Valentine, 2012, «La demeure parisienne et le travail des limites au Moyen Âge et au début des temps modernes », dans La demeure médiévale à Paris, p. 3845

ESCHER Noémie, 2009, Recueil des chartes de l'abbaye Sainte-Geneviève de Paris ([9961016]-1200), Thèse de l'École nationale des chartes.

ETIENNE Geneviève, 1974, Étude topographique sur les possessions de la maison du Temple à Paris (XII ${ }^{e}-X I V^{e}$ siècles), Thèse pour le diplôme d'archiviste paléographe

1975, «La Villeneuve du Temple à Paris aux XIII ${ }^{\mathrm{e}}$ et XIV ${ }^{\mathrm{e}}$ siècles », dans Actes $d u 100^{e}$ Congrès des Sociétés savantes, Paris, Philologie et histoire, t. II, p. 87-99

FRANKLIN Alfred, 1874 [reprint : 1984], Les rues et les cris de Paris au XIII siècle, Paris, Les Éditions de Paris

Guerard Benjamin, 1850, Cartulaire de l'église Notre-Dame de Paris, 4 vol., Paris, Imprimerie de Crapelet (Collection des cartulaires de France)

Guerber Estelle, 2001, Le quartier de l'hôtel Saint-Paul à Paris (1360-1550). Étude topographique, économique et sociale, Thèse de l'École nationale des chartes. http://www.encsorbonne.fr/fr/positions-these/quartier-hotel-saint-paul-paris-1360-1550

HAMON Etienne et WeISs Valentine (dir.), 2012, La demeure médiévale à Paris, Paris, Somogy éditions d'art, Archives nationales

HAMON Etienne, 2012, «Opérations urbaines, lotissements et habitat sur les ponts à la fin du Moyen Âge », dans La demeure médiévale à Paris, p. 50-55

Hillairet Jacques, 1963, Dictionnaire historique des rues de Paris, 2 vol., Paris, Les éditions de minuit

Leguay Jean-Pierre, 1984, La rue au Moyen Âge, Rennes, Ouest-France

MAREuSE Edgar (éd.), 1875, Le dit des rues de Paris par Guillot (de Paris); avec préface, notes et glossaire, suivi d'un plan de Paris sous Philippe le Bel, Paris, Librairie générale

MERCIER Alain, 2012, La deuxième fille de Cluny. Grandeurs et misères de Saint-Martin-desChamps, Paris, Glénat, Le Cnam

MERCIER Louis-Sébastien, 1781-1788 [reprint: 2006], Le tableau de Paris, Paris, La Découverte

NiCOLAs Catherine (éd.), 2012, Le Dit des rues de Paris : texte revu à partir du manuscrit BN fr. 24432, Paris, Les éditions de Paris 
Poulle Emmanuel, 1996, «Classement et cotation des chartriers au Moyen Âge », dans La conservation des manuscrits et des archives au Moyen Âge. XI colloque du CIPL, Scriptorium, 50-2, p. 344-355

Roux Simone, 1989, Le quartier de l'université à Paris du XIII ${ }^{e}$ au XV siècle : étude urbaine, 4 t., Thèse de Doctorat d'État, Paris, Université Paris X-Nanterre

SUMPF Alexandre, 1998-1999, Le quartier Saint-Paul à la fin du XIII siècle. La censive SaintEloi dans la paroisse Saint-Paul, 1280-1302, Mâtrise de l'Université Paris I

TERroine Anne et FossiER Lucie, Chartes et documents de l'abbaye de Saint-Magloire, 3 vol.,Turnhout, Brepols, Paris, CNRS éditions.

1998a, Chartes et documents de l'abbaye de Saint-Magloire, t. 1

1966, Chartes et documents de l'abbaye de Saint-Magloire, t. 2

1976, Chartes et documents de l'abbaye de Saint-Magloire, t. 3 $181-208$

1998b, «Le chartrier de l'abbaye Saint-Magloire de Paris », dans Revue Mabillon, p.

ThOMAs Nicolas, Bourlet Caroline, Roms Cédric, mai-juin-juillet 2007, « La Villeneuve du Temple », dans Histoire et images médiévales, $\mathrm{n}^{\circ}$ 9, Paris au Moyen Âge, p. 60-67

WEISS Valentine, 1993, Étude de topographie parisienne : la censive de Saint-Martin-desChamps à Paris du XIII siècle au début $d u X V^{e}$ siècle, Thèse pour le diplôme d'archiviste paléographe

2009, Cens et rentes à Paris au Moyen Âge. Documents et méthodes de gestion domaniale, 2 vol., Paris, Editions Honoré Champion (Histoire et archives) 